\title{
Inhibited and impulsive subgroups of socially anxious young adults: Their depressive symptoms and life satisfaction
}

\author{
Maria Tillfors ${ }^{1}$, Ewa Mörtberg ${ }^{2}$, Nejra Van Zalk ${ }^{3}$, Margaret Kerr ${ }^{3^{*}}$ \\ ${ }^{1}$ Center for Health and Medical Psychology, Institution of Law, Psychology and Social Work, Örebro University, Örebro, Sweden \\ ${ }^{2}$ Department of Psychology, Stockholm University, Stockholm, Sweden \\ ${ }^{3}$ Center for Developmental Research, Institution of Law, Psychology and Social Work, Örebro University, Örebro, Sweden \\ Email: maria.tillfors@,oru.se
}

Received 10 January 2013; revised 9 February 2013; accepted 18 February 2013

\begin{abstract}
Purpose: Socially anxious people are typically thought of as being behaviorally inhibited; however, an atypical subgroup, which is impulsive rather than inhibited, has recently been identified [1]. Theoretically, inhibition and impulsivity could be viewed as different strategies for coping with anxiety that have the same goal-escape from negative emotions-but they seem to have different implications. Previous studies have found that the socially anxious-impulsive subgroup was higher on risk-prone behavior, as for example drug use, compared with a socially anxiousinhibited subgroup [1]. In this study, we aimed to identify these subgroups in a general population, and asked whether they also experience various levels of depressive symptoms and life satisfaction, as well as moderating effects of gender. Methods: Cluster analysis was used to identify subgroups of young adults (20 - 24 years old; $N=772$ ) characterized by different profiles of social anxiety and impulsivity. These subgroups were compared on levels of internal adjustment, and the moderating effects of gender were also tested. Results: We identified five clusters, including an Anxious-Inhibited and an Anxious-Impulsive cluster. In the interaction between gender and cluster membership, gender showed evidence of moderation regarding both depressive symptoms and life satisfaction, with the young women in the Anxious-Inhibited and the Anxious-Impulsive clusters faring worst. Conclusions: We replicated previous findings demonstrating the existence of a socially anxious-impulsive subgroup, thus solidifying current knowledge that may be important when it comes to diagnostics and treatment. This may prove particularly important for young women regarding internalizing symptoms.
\end{abstract}

\footnotetext{
${ }^{*}$ This article is in memory of Professor Margaret Kerr who sadly passed
} away in late 2012
Keywords: Social Anxiety; Impulsivity; Depressive Symptoms; Life Satisfaction; Young Adults

\section{INTRODUCTION}

Socially anxious people are typically thought of as being behaviorally inhibited and avoiding anxiety-provoking social situations. Although the majority of socially anxious people fit this description, an unexpected or atypical subgroup has recently been identified. Rather than being inhibited, this subgroup of socially anxious people appear to be impulsive [1-3]. What could be the reasons for this atypical pattern of characteristics, and what does it imply for the life experiences and adaptation of socially anxious individuals?

Theoretically, inhibition and impulsivity can be seen as different strategies for coping with social anxiety. These strategies have the same function-allowing the person to escape from unpleasant inner experiences such as intense negative emotions. According to Kashdan and colleagues [1], the impulsive strategy might occur for socially anxious people who regularly expose themselves to highly anxiety-provoking situations, thus exhausting their ability to control the natural tendency to escape [see 4]. The result is an increase in automatic, impulsive behavior. If this is the case, it could have implications for different forms of adaptation. The implications for external adjustment among socially anxious-impulsive people have been shown for risk-prone behaviors such as unsafe sex and drug use [1-3], but internal adjustment might be affected as well.

One form of internal adaptation that might be affected by socially anxious-impulsive behavior is the development of depressive symptoms. Anxious-inhibited behavior has been linked to depression $[5,6]$, and the link might be as strong for anxious-impulsive behavior because of a similar mechanism-rumination. Those who are anxious-inhibited tend to overtly avoid social situa- 
tions, and avoidance tends to prompt thought processes such as rumination about what others will think when the person never shows up at social gatherings. Indeed, rumination is generally high among socially anxious people [7]. Rumination is also associated with depression. Those who are anxious-impulsive might also tend to ruminate about how their behaviors are perceived by others, but in their case it would be impulsive behavior, such as intoxication and impulsive sexual behavior. By this reasoning, one could expect high levels of depressive symptoms among the anxious-impulsive as well as the anxious-inhibited subgroups. Nevertheless, a study using a clinical sample of individuals with social anxiety disorder has not found links to co-morbid mood disorder for the anxious-impulsive subgroup [1].

One consequence of research on categorical diagnoses according to the DSM is the lack of possibility in capturing the entire picture of mental problems. Instead, the information is often restricted to the development of extreme, or clinical levels of for example anxiety and depressive symptoms, more or less based on arbitrarily set "cut-offs". This categorical classification system does therefore not fully reflect the dimensionality of mental problems [7]. It is therefore important to examine if we could replicate the previous findings of an anxious-impulsive subgroup, and identify whether this subgroup is associated with higher levels of depressive symptoms than those who are not socially anxious in a general population as well. To our knowledge, this has not yet been done in the current literature.

Another form of internal adaptation that might be affected by atypical, socially anxious-impulsive behavior is life satisfaction. Considered a cognitive aspect of subjective well-being, life satisfaction reflects an overall assessment people make of their lives. It does not necessarily correspond to symptoms such as anxiety or depression [8], thus making it significant in itself. Although social anxiety has been linked to low levels of life satisfaction [9], little is known about whether and how the various subgroups of people with social anxiety differ on well-being. Hence, the identification of socially anxious subgroups and their levels of depressive symptoms and life satisfaction would yield a better overall picture of these individuals' adjustment.

Different forms of social anxiety and their implications for depressive symptoms and life satisfaction are particularly relevant in young-adult populations. For young adults, establishing new social networks is a timely issue, and socially anxious young adults might be particularly susceptible to exhausting their abilities to control the escape tendency. Thus, they might be prone to impulsive behavior. In addition, socially anxious young adults have been shown to have few and low quality interpersonal relationships, and they tend to hesitate about pursuing goals such as education because of their social anxiety [6]. Thus, young adults with social anxiety should show lower life satisfaction than young adults without social anxiety. The question is how the typical, anxious-inhibited subgroup might compare with an atypical, anxious-impulsive subgroup in young adulthood.

The main objective of the current study is to distinguish profiles of social anxiety and impulsivity among young adults in a general population, and compare them on internal adjustment. We aimed to identify an atypical, anxious-impulsive subgroup as well as a typical, anxious-inhibited subgroup, comparing them on levels of depressive symptoms and life satisfaction. In addition, as earlier research has shown that both internalizing [6] and externalizing problems [10] might have different consequences for women than for men, we examined the possible moderating effects of gender on these associations. In short, our questions were as follows: 1) Can anxiousimpulsive and anxious-inhibited subgroups be identified in a general population of young adults? and if so 2) are anxious-impulsive and anxious-inhibited subgroups higher on depressive symptoms and lower on life satisfaction than those who are not socially anxious and do they differ from each other? and c) are these associations moderated by gender?

\section{MATERIALS AND METHODS}

\subsection{Participants and Procedure}

The participants were from a community in Western Europe with a total population of about 26,000. At the time of the data collection, the unemployment rate in the community $(11 \%)$ was slightly higher than the rest of the country $(7 \%)$. The percentage of immigrants (16\%) was comparable to the rest of the country $(15 \%)$. Mean incomes were about $7.0 \%$ lower than in the rest of the country. The data collection was aimed at young adults who were $20-24$ years old. After receiving a postcard with information about the study, the participants were sent questionnaires asking them about social anxiety and other related issues. A lottery ticket was included with each questionnaire. Because this age group is notoriously difficult to reach in survey research, telephone interviews were conducted with those who were willing to participate, but did not want to fill out questionnaires. An abbreviated form of the questionnaire was adapted for the telephone interviews, but all measures used in the current study were included. The final sample comprised 772 participants (450 women and 322 men; Mage = 22.09). The participants were informed about the questions and the ethical aspects of the study through a standardized procedure. The procedures and measures were approved by the Regional Research Ethics Committee. 


\subsection{Measures}

\subsubsection{Social Anxiety}

Social anxiety was measured with the Social Phobia Screening Questionnaire (SPSQ), which includes 14 questions about distress in different social situations [11]. Examples of items were "Speaking or performing in front of a group," "Expressing your own opinions in front of others," "Calling someone you do not know very well". The participants rated themselves on a five-point scale with the response options: Not at all distressing (1), Somewhat distressing (2), Moderately distressing (3), Very distressing (4), and Extremely distressing (5). The Cronbach's alpha for this scale was 0.90 .

In a validation study using a diagnostic interview as a reference, the sensitivity of the SPSQ was found to be $100 \%$ and the specificity $95 \%$ [11]. Lastly, this 14 -item distress scale has been found to correlate highly with two well-established social phobia scales, the Social Phobia Scale, $r=0.77$, and the Social Interaction Anxiety scale, $\mathrm{r}=0.79[11,12]$, which indicates an adequate concurrent validity for this scale.

\subsubsection{Impulsivity}

The measure of impulsivity was taken from a larger instrument created to assess psychopathy-like personality traits among adolescents in community samples [13]. The impulsivity subscale comprised 5 items, rated on a four-point scale with the response options: Does not apply at all (1), Applies poorly (2), Applies quite well (3) and Applies very well (4). Examples of items were: "You prefer to spend your money right away rather than saving it," "You consider yourself a rather impulsive person," and "You often do things without thinking ahead." The Cronbach's alpha for this scale was 0.62 .

\subsubsection{Depressive Symptoms}

Depressive symptoms were measured with a shortened version of the Child Depression Scale from the Center for Epidemiological Studies, which assesses depressive symptoms such as worry, sadness, hopelessness, lethargy, and poor appetite [14]. The shortened version included 7 of the original questions, based on a factor analysis conducted on the entire scale [15]. The response items were: Not at all (1), Occasionally (2), From time to time (3), and Often (4). Participants were instructed to think about the past week. Examples of items were: "How often have you worried about things you don't usually worry about," "How often have you felt down and unhappy," and "How often have you felt sad?" The Cronbach's alpha was 0.89 .

\subsubsection{Life Satisfaction}

Life satisfaction was measured using 5 items from the
Satisfaction with Life Scale [16]. Examples of items were: "You are satisfied with your life," "Up until today, you have achieved what you think is important in life," and "If you could relive your life, you would hardly change anything." The response options were: Not at all true (1), Not true (2), Not quite true (3), Neither true nor untrue (4), Partly true (5), True (6), and Completely true (7). The response options were reduced for the telephone interview, because shorter response scales have been shown to correspond with more reliable answers during telephone interviews [17]. The response items used during the telephone interview were: Not at all true (1), Not quite true (2), Neither true nor untrue (3), Partly true (4), and Completely true (5). The response options from the written questionnaires were re-coded so as to match the response options from the telephone interviews. Options 1 and 2 became option 1 (Not at all true); option 3 became option 2 (Not quite true); option 4 became option 3 (Neither true nor false); option 5 became option 4 (Partially true), and options 6 and 7 became option 5 (Completely true). The Cronbach's alpha was 0.90 . The correlation between life satisfaction and depression was -0.36 .

\subsection{Analyses}

Cluster analysis using SPSS was used to classify participants into different profiles of social anxiety and impulsivity. All of the variables were Z-transformed before being entered into the analyses. To answer our research questions about the links between social anxiety, impulsivity, depressive symptoms, and life satisfaction, twoway (Gender $\times$ Cluster) factorial analyses of variance (ANOVAs) with post hoc tests were conducted, with depressive symptoms and life satisfaction as outcome variables.

\section{RESULTS}

\subsection{Can Anxious-Impulsive and Anxious-Inhibited Subgroups Be Identified in a General Population of Young Adults?}

To identify homogeneous subgroups of young adults with social anxiety and impulsivity, we conducted hierarchical cluster analyses using Ward's method and squared Euclidean distances. There are several criteria and guidelines for the number of clusters to be selected, but currently no selection procedure is generally believed to be more beneficial than others. Our final choice of cluster solution was based on a combination of the following four recommended criteria: 1) the cluster solution should be theoretically meaningful $[18,19] ; 2)$ clusters are considered reasonably homogenous with Explained Error Sums of Squares (EESS) values ideally around $67 \%$ and not less than $50 \%$ [18]; 3) the cluster coefficient per- 
centage change to the next level should preferably not be less than $10 \%$ [19], and 4) each cluster should contain at least 10 individuals [19].

Table 1 shows the EESS-values and the change in percentage of the cluster coefficient to the next level for the cluster solutions between 3 to 10 clusters. As can be seen in the table, and based on the second abovementioned criteria about the EESS-value being above 67\%, all of the cluster solutions between 5 to 10 clusters could be used. Regarding the cluster coefficient percentage change, all of the cluster solutions were above the recommended value of $10 \%$, which fulfilled the third criteria. In addition, the fourth criteria were also obtained, as the smallest cluster comprised 12 people, which was higher than the optimal limit of 10 . Nonetheless, and as specified in the first criteria, the theoretical meaningfulness of a cluster solution of 10 clusters was questionable. The patterns that emerged in a 5-cluster solution were recurrent for all other cluster solutions, as only the size of the variables and the number of clusters varied overall. Hence, to choose a solution with more than five clusters did not add anything of theoretical value above and beyond a 5-cluster solution. Thus, due to fulfilled criteria and theoretical meaningfulness, the 5-cluster solution was used as a final choice in further analyses.

The final clusters are shown in Table 2. As can be seen in the table, we identified an Anxious-Impulsive cluster (those high on social anxiety and impulsivity), comprising the smallest number of people (8\%), which was expected. An Anxious-Inhibited cluster (10\%) and an Impulsive cluster $(26 \%)$ were also identified. These clusters comprised young adults high on social anxiety or impulsivity, respectively. Finally, two clusters we termed No Problems (27\%) and No Problems/Low (29\%) were also identified. These included young adults who were

Table 1. Explained error sums of squares (EESS-values) for the 3 - 10 cluster solutions with the change in percentage for the cluster coefficients.

\begin{tabular}{ccccccccc}
\hline Cluster solution & 3 & 4 & 5 & 6 & 7 & 8 & 9 & 10 \\
EESS (\%) & 56 & 64 & 69 & 73 & 76 & 79 & 81 & 83 \\
Coefficient (\%) & 57 & 20 & 20 & 13 & 13 & 14 & 12 & 13 \\
\hline
\end{tabular}

Table 2. Means (standard deviations) for the 5-cluster solution.

\begin{tabular}{cccc}
\hline Clusters & Social Anxiety & Impulsivity & $N($ Men $)$ \\
\hline Anxious-Impulsive & $1.32(0.83)$ & $0.82(0.46)$ & $63(26)$ \\
Anxious-Inhibited & $1.94(0.82)$ & $-0.69(0.61)$ & $73(34)$ \\
Impulsive & $-0.51(0.51)$ & $0.97(0.61)$ & $201(85)$ \\
No Problems & $0.15(0.43)$ & $-0.36(0.49)$ & $208(88)$ \\
No Problems/Low & $-0.67(0.44)$ & $-0.93(0.52)$ & $227(89)$ \\
\hline
\end{tabular}

high on neither social anxiety nor impulsivity, with the No Problems/Low cluster being particularly low on these measures. In sum then, we identified an atypical, socially anxious-impulsive cluster, along with a more typical, socially anxious-inhibited cluster in a general population of young adults. These findings support previous work using clinical samples [see e.g., 1].

\subsection{Are Anxious-Impulsive and Anxious-Inhibited Clusters Higher on Depressive Symptoms and Life Satisfaction than the Other Clusters and Do They Differ from Each Other?}

We compared young adults in all of the clusters on depressive symptoms and life satisfaction, using two-way (Gender $\times$ Cluster solution) factorial ANOVAs with Hochberg's post hoc tests. The means and group differences are shown in Table 3. The overall models were significant for both of the outcome variables $(\mathrm{F}(9768)=15.49$, $\mathrm{p}<0.001$ for depressive symptoms and $\mathrm{F}(9768)=9.50$, $\mathrm{p}<0.001$ for life satisfaction). The main effect of gender was significant regarding depressive symptoms $(\mathrm{F}(1768)$ $=43.61, \mathrm{p}<0.001)$. The main effect of cluster was also significant in both models $(\mathrm{F}(4768)=22.37, \mathrm{p}<0.001$ for depressive symptoms and $\mathrm{F}(4768)=15.63, \mathrm{p}<0.001$ for life satisfaction). As can be seen in Table 3, the post hoc tests show that people in the Anxious-Impulsive and Anxious-Inhibited clusters faring worse than those in the other clusters both when it comes to depressive symptoms and life satisfaction. Hence, in general AnxiousImpulsive and Anxious-Inhibited clusters are higher on depressive symptoms and lower on life satisfaction than the other clusters but they do not differ from each other.

Table 3. Mean depressive symptoms and life satisfaction by cluster from two-way ANOVAs with Hochberg's post hoc tests.

\begin{tabular}{lc}
\hline Cluster & Mean $(S D)$ \\
\hline & Depressive Symptoms \\
Anxious-Impulsive (a) & $2.29^{\text {cde }}(0.81)$ \\
Anxious-Inhibited (b) & $2.26^{\text {cde }}(0.72)$ \\
Impulsive (c) & $1.80^{\text {abe }}(0.71)$ \\
No Problems (d) & $1.76^{\text {abe }}(0.68)$ \\
No Problems/Low (e) & $1.58^{\text {abcd }}(0.59)$ \\
& $2.88^{\text {de }}(1.22)$ \\
Anxious-Impulsive (a) & $2.72^{\text {cde }}(1.21)$ \\
Anxious-Inhibited (b) & $3.29^{\text {be }}(1.14)$ \\
Impulsive (c) & $3.43^{\text {abe }}(1.10)$ \\
No Problems (d) & $3.77^{\text {abcd }}(1.11)$ \\
No Problems/Low (e) &
\end{tabular}

Note: Superscript letters indicate which cluster means differed from the current mean at $\mathrm{p}<0.05$ or below according to Hochberg's post hoc tests. 


\subsection{Are These Associations Moderated by Gender?}

In the Gender $\times$ Cluster solution interactions, gender showed evidence of moderation regarding both depressive symptoms and life satisfaction $(\mathrm{F}(4768)=2.02, \mathrm{p}=$ 0.09 for depressive symptoms and $\mathrm{F}(4768)=2.65, \mathrm{p}<$ 0.05 for life satisfaction). For that reason, we ran ANOVAs with Hochberg's post hoc tests separately for men and women. The overall model for depressive symptoms was significant for both genders $(\mathrm{F}(4446)=$ $19.98, \mathrm{p}<0.001$ for women and $\mathrm{F}(4322)=5.78, \mathrm{p}<$ 0.001 for men, respectively). This was also true for life satisfaction $(\mathrm{F}(4446)=10.55, \mathrm{p}<0.001$ for women and $\mathrm{F}(4322)=7.98, \mathrm{p}<0.001$ for men, respectively). The cluster means and post hoc tests are shown in Table 4. As the table shows, it seems that the Anxious-Impulsive and Anxious-Inhibited women were worse off than the men, as they had significantly higher means on depressive symptoms and significantly lower means on life satisfaction than all of the other clusters. Generally speaking, the socially anxious women-regardless of their levels of inhibition or impulsivity-seemed to fare worse than the men on both depressive symptoms and life satisfaction.

In sum, gender showed evidence of moderation between cluster membership and both depressive symptoms and life satisfaction, with women in the AnxiousImpulsive and Anxious-Inhibited clusters faring worst.

\section{DISCUSSION}

Previous research has identified subgroups of anxiousimpulsive and anxious-inhibited young adults, and com-

Table 4. Cluster means (SDs) on depressive symptoms and life satisfaction from ANOVAs calculated separately for men and women.

\begin{tabular}{ccc}
\hline Clusters & Women Mean (SD) & Men Mean (SD) \\
\hline \multicolumn{3}{c}{ Depressive Symptoms } \\
Anxious-Impulsive (a) & $2.56^{\text {cde }}(0.72)$ & $1.92^{\mathrm{e}}(0.79)$ \\
Anxious-Inhibited (b) & $2.51^{\text {cde }}(0.67)$ & $1.98^{\text {de }}(0.67)$ \\
Impulsive (c) & $1.90^{\text {abe }}(0.77)$ & $1.66(0.60)$ \\
No Problems (d) & $1.87^{\text {ab }}(0.71)$ & $1.61^{\mathrm{b}}(0.61)$ \\
No Problems/Low (e) & $1.66^{\text {abc }}(0.59)$ & $1.46^{\text {ab }}(0.58)$ \\
& Life Satisfaction & \\
Anxious-Impulsive (a) & $2.74^{\text {cde }}(1.25)$ & $3.08(1.17)$ \\
Anxious-Inhibited (b) & $2.78^{\text {cde }}(1.27)$ & $2.65^{\mathrm{e}}(1.15)$ \\
Impulsive (c) & $3.48^{\text {ab }}(1.18)$ & $3.04^{\mathrm{e}}(1.06)$ \\
No Problems (d) & $3.66^{\mathrm{ab}}(1.10)$ & $3.12^{\mathrm{e}}(1.04)$ \\
No Problems/Low (e) & $3.78^{\mathrm{ab}}(1.08)$ & $3.74^{\text {bcd }}(1.17)$ \\
\hline
\end{tabular}

Note: Superscript letters indicate which cluster means differed from the current mean at $\mathrm{p}<0.05$ or below according to Hochberg's post hoc tests. pared them regarding external adjustment [1-3]. Going beyond previous research, however, we attempted to identify these subgroups in a general population, compare them on levels of internal adjustment, as well as examine if these associations were moderated by gender. We found that gender showed evidence of moderation in these associations, as the young women in the anxiousimpulsive and anxious-inhibited subgroups fared worse than the men. We did not, however, find differences on depressive symptoms or life satisfaction between individuals who were anxious-impulsive and anxious- inhibited.

As for the anxious-inhibited subgroup, our results are consistent with previous research showing positive relations between social anxiety and depression, as well as negative relations between social anxiety and life satisfaction [6,9]. The central dilemma of social anxiety can be viewed as an approach-avoidance conflict between wanting to take part in social interactions and, at the same time, wanting to avoid negative emotions associated with approaching others [20]. If we interpret our results in light of an approach/avoidance conflict, it seems that the fear and avoidance of an adverse outcome in social situations may trump the possibility of any reward gained from approach behavior among socially anxious-inhibited individuals. Because interpersonal relationships are one of the main sources of life satisfaction [21], and the anxious-inhibited group differed from all except the anxious-impulsive group regarding both depressive symptoms and life satisfaction, our results seem plausible.

Knowledge about the anxious-impulsive subgroup regarding the approach-avoidance conflict is sparse, nonetheless. One could imagine that approach behavior and participation in social situations should be greater among people in the anxious-impulsive subgroup. As a result, anxious-impulsive people may take part in social situations to a greater extent than anxious-inhibited people. However, using control strategies with the function of avoiding negative emotions has in the long term been linked to low positive affect [e.g., 22]. This does not imply that people in the anxious-impulsive group experience more positive emotions in general. Indeed, this did not seem to be the case in the current study, as the anxious-impulsive group fared as poorly as the anxiousinhibited group in terms of both depressive symptoms and life satisfaction. Unfortunately, we cannot differentiate between short and long term implications of our results. However, based on the same type of classification we have used in this study, Kashdan and Weeks showed that the anxious-impulsive group reported short-term pleasure of impulsive behavior in social situations, such as increased sexual activity and social proximity [23]. If the same were true in this sample, those feelings were 
not transformed into a more global sense of life satisfaction. A possible mechanism explaining why these people fail to transform the short-term pleasure to a sense of life satisfaction could be that their memories of social situations are mostly negatively colored in retrospect, and they may, therefore, try to avoid these unpleasant inner experiences by using such control strategies as worry and rumination [23]. This notion is in line with the fact that emotional control strategies may work in the short term by relieving anxiety, but in the long term they often fail and are associated with the maintenance of ill-health [7]. In addition, this could also be one factor that explains why young women in this study fared worst, because women more than men use worry and rumination as strategies to regulate emotions [7]. Hence, these two explanations should be interpreted cautiously, and further research is needed to empirically test them.

In our results, the women in the anxious-impulsive and anxious-inhibited groups fared worse than men, which is in accordance with previous research where the gender ratio of depressive symptoms was twice as high for adolescent girls as for boys [24]. Trying to regulate emotions by impulsive behaviors, such as getting intoxicated at a party or engaging in impulsive sexual acts may come at a higher cost for women than men. In our study, we found support for this idea, as there was a significant difference between women and men in the anxious-impulsive group regarding depressive symptoms.

This study has several limitations. First, we only used self-report measures. Nonetheless, previous research has demonstrated that people's own reports of internalizing problems quite accurately measure subjective experiences [25]. Second, we used dimensional measures of symptoms. Even though the dimensional view is assumed to be a better measure of elevated symptomatology that later proceeds on to clinical levels than the categorical clinical view is, the dimensional approach could yield different results than a clinical view. Third, the reliability of the impulsivity measure was not as high as the other measures. This might be because it is difficult for people to judge their own impulsivity. Evidence for convergent and discriminant validity of the scale has been found, however, as it loads on the correct factor in the overall instrument and does not cross-load on the other factors [13]. Nonetheless, the relatively low reliability might have made our results more conservative than they would have been if the measure had been more internally consistent.

Despite these limitations, our study has several strengths. First, we used both variable- and person-oriented approaches to answer our research questions [18]. Second, except for the measure of impulsivity, all of the scales used in the study showed good psychometric properties. Third, we also used dimensional instead of categorical measures, which increases the variance and thus the power in our analyses. Finally, we used measures of both negative and positive affect in the same study, which has seldom been done in previous research. Thus, this study extends previous findings to a normal population, and revealing that gender showed evidence of moderation regarding both depressive symptoms and life satisfaction, with the young women in the AnxiousInhibited and the Anxious-Impulsive clusters faring worst.

\section{CONCLUSION}

To conclude, our results indicate that increased awareness is needed regarding subgroups of social anxiety in general populations, and particularly a socially anxious-impulsive subgroup of individuals. This combination appeared to be particularly hazardous for young women, who showed the highest levels of internalizing problems in the current study. This information could be another essential piece in the social anxiety subgroup puzzle that could prove important in the prevention of maladjustment for socially anxious individuals.

\section{ACKNOWLEDGEMENTS}

This research was funded by a grant from the Swedish Research Council to Maria Tillfors, Nejra Van Zalk, and Margaret Kerr. The longitudinal study was funded by grants from the Swedish Research Council. We acknowledge the support of the teachers, principals, and pupils who participated in the " 10 to 18 " Study.

\section{REFERENCES}

[1] Kashdan, T.B., McKnight, P.E., Richey, J.-A., et al. (2009) When social anxiety disorder co-exists with risk-prone, approach behavior: Investigating a neglected, meaningful subset of people in the National Comorbidity Survey-Replication. Behaviour Research and Therapy, 47, 559-568. doi:10.1016/j.brat.2009.03.010

[2] Kashdan, T.B., Elhai, J.D. and Breen, W.E. (2008) Social anxiety and disinhibition: An analysis of curiousity and social rank appraisals, approach-avoidance conflicts, and disruptive risk-taking behavior. Journal of Anxiety Disorders, 22, 925-939. doi:10.1016/j.janxdis.2007.09.009

[3] Kashdan, T.B. and Hofmann, S.G. (2008) The high-novelty-seeking, impulsive subtype of generalized social anxiety disorder. Depression and Anxiety, 25, 535-541. doi:10.1002/da.20382

[4] Baumeister, R.F., Gailliot, M., DeWall, C.N., et al. (2006) Self-regulation and personality: How interventions increase regulatory success, and how depletion moderates the effects of traits on behavior. Journal of Personality, 74, 1773-1801. doi:10.1111/j.1467-6494.2006.00428.x

[5] Wittchen, H.-U., Beesdo, K., Bittner, A., et al. (2003) Depressive episodes - Evidence for a causal role of primary anxiety disorders? European Psychiatry, 18, 384-393. 
doi:10.1016/i.eurpsy.2003.10.001

[6] Rapee, R.M. and Spence, S.H. (2004) The etiology of social phobia: Empirical evidence and an initial model. Clinical Psychology Review, 24, 737-767. doi:10.1016/j.cpr.2004.06.004

[7] Harvey, A., Watkins, E., Mansell, W., et al. (2004) Cognitive behavioral processes across psychological disorders: A transdiagnostic approach to research and treatment. Oxford University Press Inc., New York.

[8] Pavot, W. and Diener, E. (1993) Review of the satisfaction with life scale. Psychological Assessment, 5, 164-172. doi:10.1037/1040-3590.5.2.164

[9] Barrera, T.L. and Norton, P.J. (2009) Quality of life impairment in generalized anxiety disorder, social phobia, and panic disorder. Journal of Anxiety Disorders, 23, 1086-1090. doi:10.1016/j.janxdis.2009.07.011

[10] Moffitt, T.E. and Caspi, A. (2001) Childhood predictors differentiate life-course persistent and adolescence-limited antisocial pathways among males and females. Development and Psychopathology, 13, 355-375. doi:10.1017/S0954579401002097

[11] Furmark, T., Tillfors, M., Everz, P.O., et al. (1999) Social phobia in the general population: Prevalence and sociodemographic profile. Social Psychiatry and Psychiatric Epidemiology, 34, 416-424. doi:10.1007/s001270050163

[12] Heimberg, R.G., Mueller, G.P., Holt, C.S., et al. (1992) Assessment of anxiety in social interaction and being observed by others: The social interaction anxiety scale and the social phobia scale. Behavior Therapy, 23, 53-73. doi:10.1016/S0005-7894(05)80308-9

[13] Andershed, H., Kerr, M., Stattin, H., et al. (2002) Psychopathic traits in non-referred youths: A new assessment tool. In: Blaauw, E. and Sheridan, L., Eds., Psychopaths: Current International Perspectives, Elsevier, Hague, 131158.

[14] Radloff, L.S. (1977) The CES-D scale: A self-report depression scale for research in the general population. Applied Psychological Measurement, 1, 385-401. doi:10.1177/014662167700100306

[15] Olsson, G. and von Knorring, A.-L. (1997) Depression among Swedish adolescents measured by the self-rating scale center for epidemiology studies-Depression child (CES-DC). European Child and Adolescent Psychiatry, 6,
81-87.

[16] Diener, E., Emmons, R.A., Larsen, R.J., et al. (1985) The satisfaction with life scale. Journal of Personality Assessment, 49, 71-75. doi:10.1207/s15327752jpa4901_13

[17] Japec, L., Ahtiainen, A., Hörngren, J., et al. (1997) Minska bortfallet. Statistics, Sweden.

[18] Bergman, L.R., Magnusson, D. and El-Khouri, B.M. (2003) Studying individual development in an interindividual context: A person-oriented approach. In: Magnusson, D., Ed., Paths through Life, Vol. 4, Psychology Press, New York.

[19] Hair, J.F., Anderson, R.E., Tatham, R.L., et al. (1995) Multivariate data analysis with readings. 4th Edition, Englewood Cliffs, Prentice-Hall, Inc., Upper Saddle River.

[20] Kimbrel, N.A., Mitchell, J.T. and Nelson-Gray, R.O. (2010) An examination of the relationship between behavioral approach system (BAS) sensitivity and social interaction anxiety. Journal of Anxiety Disorders, 24, 372-378. doi:10.1016/j.janxdis.2010.02.002

[21] Diener, E. and Seligman, M.E.P. (2002) Very happy people. Psychological Science, 13, 81-84. doi:10.1111/1467-9280.00415

[22] Kashdan, T.B. and Breen, W.E. (2008) Social anxiety and positive emotions: A prospective examination of a selfregulatory model with tendencies to suppress or express emotions as a moderating variable. Behavior Therapy, 39, 1-12. doi:10.1016/j.beth.2007.02.003

[23] Kashdan, T.B. and Weeks, J.W. (2010) Social anxiety, positive experiences, and positive events. In: Hofmann, S.G. and Dibartolo, P.M., Eds., Social Anxiety: Clinical, Developmental, and Social Perspectives, Elsevier Academic Press, San Diego, 447-469.

[24] Ingoldsby, E.M., Kohl, G.O., McMahon, R.J., et al. (2006) Conduct problems, depressive symptomatology and their co-occurring presentation in childhood as predictors of adjustment in early adolescence. Journal of Abnormal Child Psychology, 34, 603-621. doi:10.1007/s10802-006-9044-9

[25] Trost, K., Biesecker, G., Stattin, H., et al. (2007) Not wanting parents' involvement: Sign of autonomy or sign of problems? European Journal of Developmental Psychology, 4, 314-331. doi:10.1080/17405620601008980 